\title{
REVIEWS
} FILIP SZTEN 2 , B, C

\section{Brdt Bromodomains Inhibitors and Other Modern Means of Male Contraception}

${ }^{1}$ Department of Endocrinology, Diabetes and Radionuclide Therapy, Wroclaw Medical University, Poland

2 Students of Medical Department, Wroclaw Medical University, Poland

A - research concept and design; B - collection and/or assembly of data; $\mathbf{C}$ - data analysis and interpretation;

$\mathbf{D}$ - writing the article; $\mathbf{E}$ - critical revision of the article; $\mathbf{F}$ - final approval of article; $\mathbf{G}$ - other

\begin{abstract}
Compared to efficient and secure female contraception, a vasectomy and condoms are the only options for men. The choice of male contraceptive methods is limited, so contraception mainly rests on the shoulders of women. Several concepts are considered: testosterone administration - inhibiting pituitary secretion of lutropin (LH) and follicle stimulating hormone (FSH), progestogen - affecting the secretion of gonadotropin and gonadoliberin $(\mathrm{GnRH})$ antagonists. New potential targets for non-hormonal male contraception were discovered: glyceraldehyde-3-phosphate-dehydrogenase (GAPDHS) - specific to male germ cells and voltage-gated cation channel (CatSper). Both are responsible for sperm motility. Drugs such as thioridazine used in schizophrenia treatment and phenoxybenzamine (antihypertensive activity) exhibit a contraceptive effect. Similar action exhibits an analogue of lonidamine - adjudin and an antagonist of retinoic acid receptors (BMS-189453). Researchers are working on a contraceptive vaccine, whose active ingredient is epididymal protease inhibitor (Eppin). Another promising method acts by blocking Bromodomain testis-specific proteins (Brdt) involved in the process of spermatogenesis. JQ1-the Brdt inhibitor causes reversible infertility without affecting the endocrine signaling pathways. A recent discovery of Juno as the binding partner for Izumol identifies these proteins as the cell-surface receptor pair, essential for gamete recognition and this interaction can be inhibited by an anti-Juno monoclonal antibody. Our review shows that the situation of men can change and investigators are close to the optimal solution. In the near future men will be able to choose the best contraceptive suited to their needs (Adv Clin Exp Med 2015, 24, 4, 705-714).
\end{abstract}

Key words: contraception, hormones, testosterone.

Compared to contraception for women, which includes a number of different methods, men have access only to condoms and a vasectomy. Described studies have shown that this situation may change. Both hormonal and non-hormonal contraception ideas are recently evolving.

There are many challenges facing researchers trying to reach a specific goal in spermatogenesis, which include: 1) an incredible number of sperm produced by the fertile men - 1,000 per heartbeat, 2 ) the difficulty of fully suppressing these millions of sperm produced per day as compared to a relatively easy task of preventing the formation of one egg per month in women, 3) the fear that an impact on the production of germ cells may change the genetic material of the offspring and 4) the blood-testis barrier, which, on the one hand, prevents harmful molecules from the blood, on the other hand, makes it difficult to create drugs of pharmacokinetics proper for that tissue. Unexpected side effects, large financial and regulatory obstacles can also play a major role [1]. In addition, effectiveness, convenience and safety of existing methods of contraception made for women has set a high bar for researchers.

This places demands on male contraception, which should be 1) as effective and safe as female methods, with the ability of achieving sterility quickly with complete restoration after the withdrawal of treatment 2) free of side effects on future 
progeny 3) financially affordable and convenient in use [2].

\section{Mechanical Methods - Condoms and Vasectomy}

Since the $19^{\text {th }}$ century, condoms have been one of the oldest and most popular methods of contraception in the world. They are also one of the basic, cheapest and quite effective methods of preventing pregnancy, but most of all they protect against sexually transmitted diseases, mainly HIV (human immunodeficiency virus), and should therefore be used as a prophylactic against the transmission of these infections [3]

The first mention of the use of condom dates back to the world of the ancient Greeks and Romans, whose approach to sexuality was unhampered. However, condoms similar to those currently used appeared in the $16^{\text {th }}$ century.

As the precursor in this field is considered by an Italian anatomist and physician Gabriella Fallopia who wrote a treatise "De Morbo Gallico" ("The French Disease, or syphilis") in 1564. He offered a linen sheath, soaked in a chemical solution wrapped around the penis to protect from syphilis. Later at the time of the accession of Charles II of England, the name "condom" appeared. Apparently this name comes from a royal physician Dr. Condom, whom the king commissioned to come up with an adequate invention for his soldiers, but at the time condoms were widely available in the UK and sold on the streets by the merchants. A breakthrough came in the mid- $19^{\text {th }}$ century, when the American Charles Goodyear in 1837, created and patented the first rubber condom. After that event condoms were mass-produced. In 1916 Julius Fromm, born in Konin, a Polish Jew, patented a method for the production of latex condoms seamless, more durable, made of thinner material. In the interwar his invention was to conquer the world. Currently most commercially available condoms are made of latex. Other substances such as AT-10 resin, polyisoprene, and polyurethane are also used in manufacture.

Polyurethane can be considered a better material than latex due to the fact that it conducts heat better and is not as sensitive to heat or ultraviolet light. It has less stringent storage requirements and a longer shelf-life. It can also be used with oil-based lubricants. Polyurethane is the material with no allergenic properties and no aftertaste. However, polyurethane condoms are less flexible than latex and appear to be more vulnerable to slipping off the penis during withdrawal or cracking than those made of latex. The disadvantage of this method is a relatively high price. Pearl index is a tool to measure the effectiveness of birth control methods and values of 10-15 for condoms (comparatively for female hormonal contraception 1-3) [3].

\section{Vasectomy}

Vasectomy is for now the most reliable method of male contraception. This is the second, after condoms, available form of protection against pregnancy. Despite its high efficiency, this procedure can generate problems resulting from insufficient information given to the patient before surgery, improper performance of surgery or inadequate care of the patient after surgery period until the definitive infertility is obtained. The principle of a vasectomy is to break the continuity of the vas deferens. This can be achieved by using different techniques. Despite the differences, there is no evidence of significant superiority of one method over the other in effectiveness in preventing pregnancy [4]. Men choose vasectomies for various reasons but the decision should be well thought out. The duty of the physician is to inform the patient about the potential complications that may occur when the procedure is performed. One of the most common long-term complications after vasectomy is a scrotal pain [5]. The etiology of the pain is unclear. Some authors, on the basis of observations of vasectomized men and the control group without performed surgery, pay attention to the obstruction of the vas deferens, sperm granulomas and chronic epididymitis as aetiologic factors. [6] Another complication is spontaneous recanalization of the vas deferens in $0.03-1.2 \%$ of patients after a proved successful vasectomy [7]. After 10 years, about $2 \%$ of vasectomized men have chosen a partially reversal surgery. Approximately $60 \%$ of men after bilateral vasectomy develop antibodies against sperm [5]. On the one hand, some researchers suggest that such antibodies may lower the chances of a successful pregnancy after vasectomy reversal [8] and vasectomy should be considered irreversible. On the other hand, other researchers claim that the pregnency rate is relatively high (50-70\%) [6] and the presence of antibodies do not closely correlate with postoperative ability to conceive [9]. Patients undergoing vasectomies are advised to avoid sport activity and exercise for a given period. Semen analysis should be performed after 3 months to determine infertility. The surgery should be repeated in patients whose postvasectomy semen analysis shows motile sperm after 6 months.

\section{Male Hormonal Contraception - Principles}

The purpose of hormonal male contraception is the inhibition of spermatogenesis to achieve 
at least severe oligospermia ( $\leq 106$ sperm $/ \mathrm{mL}$ ) [10]. Both luteinizing hormone ( $\mathrm{LH})$ and follicle stimulating hormone (FSH) are necessary for the maintenance of quantitatively normal spermatogenesis and both gonadotropins need to be suppressed to impair this process. Hormonal contraception strategy consists of the inhibition of $\mathrm{LH}$ and FSH production in the pituitary gland to decrease testosterone production in the testes and ultimately impair spermatogenesis. An effective regimen of hormonal contraception should lead to a strong negative feedback inhibition of both gonadotropins. In order to avoid side effects levels of peripheral circulating, androgens should remain within the normal range with a simultaneous decrease in their levels in the testes [10]. The inhibition of gonadotropin release, and subsequent reduction of testicular testosterone level also reduces plasma concentration of androgens and thus abolishes their anabolic effects on muscle strength and libido. Testosterone blood concentrations must therefore be maintained by androgen supplementation. The most important symptoms of testosterone deficiency include the following: weight gain, lipid imbalance (HDL/LDL), abnormal glucose level. Another nuisance is acne, low libido, decreased testicular volume and mood swings. Such effects occur in a few percent of men testing hormonal contraception.

\section{Various Strategies for Hormonal Male Contraception}

\section{Testosterone Alone}

There are various methods of testosterone administration and its combinations with other substances. The variety of methods arises from the will to obtain the right balance between benefits and side-effects. The use of testosterone as a male contraceptive has been tested for the first time by the WHO in 1996. The intramuscular administration of $200 \mathrm{mg}$ of testosterone reached full effectiveness of contraceptive method among $137 \mathrm{cou}$ ples. There was only 1 pregnancy during the efficacy phase [11]. The results of WHO research indicate the effectiveness of testosterone monotherapy in $91 \%$ Asian and only in $60 \%$ Caucasian [11]. The activity of alpha-5-reductase or polymorphism of UDP-glucuronosyltransferase, responsible for the glucuronidation of testosterone to testosterone glucuronide, may explain race-related differences. In another trial there is information about androgen receptor polymorphism that contributes to ethnic differences in response to hormonal contraceptive for men but large-scale multi-ethnic studies must be conducted to confirm this information [12]

\section{Testosterone with Gonadotropin-Suppressing Agent}

Another possibility is the administration of testosterone with progestin. This is a highly developed form of hormonal regulation of spermatogenesis in the context of male contraception. Progestin has anti-gonadotropic activity and supports the action of testosterone. The combination of these two hormones is much more effective in suppressing sperm production in Caucasians than testosterone alone [13]. Several combinations of various progestins and derivatives of testosterone have been tested in clinical trials within the last 30 years achieving different effects. It has been shown that progestins with a higher intrinsic androgenic activity are more effective in inhibiting the production of LH in rats than pure antagonists [14]. The study also demonstrated effective combination of a GnRH antagonist plus testosterone as a male contraception [15]. This antagonist acts by competitive inhibition of the action of endogenous GnRH.

\section{GnRH Agonists and Antagonists}

The GnRH agonists used alone effectively suppress testosterone levels to castrate levels in patients with prostate cancer, while suppressing spermatogenesis. One study of male contraception showed that additional testosterone supplementation paradoxically weakens $\mathrm{GnRH}$ agonist inhibitory effects on spermatogenesis, precluding the use of this combined therapy. The reason for this phenomenon is not clearly elucidated but it is suspected that the return of FSH secretion [16] and residual testosterone secretion in the testicles can restore spermatogenesis.

Another contraceptive method turned out to be ineffective, and, therefore, much has been expected from GnRH agonists. Testosterone with $\mathrm{GnRH}$ antagonists has proven much more promising. Better results can be obtained if the dose of testosterone is low, and its administration delayed [17]. GnRH antagonists do not cause any side effects other than skin reactions due to histamine release. Currently, efforts are under way to develop this method, but it will be difficult because of their high cost, difficulty of administration, low potency and local irritation [17]. 


\section{Selective Androgen Receptor Modulators}

Another possibility is the use of SARMs (Selective androgen receptor modulators). The 7a-Methyl-19-Nortestosteron (MENT) implants in combination with etonogestrel effectively suppress spermatogenesis. However, adverse reactions, including increase in haemoglobin level, an elevated systolic blood pressure and decrease in HDL cholesterol. This promising result was not sustained due to a decline in MENT release from the implants [18].

\section{Dimethandrolone Undecanoate}

Dimethandrolone undecanoate (DMAU: $17 \beta$ -undecanoate $7 \alpha, 11 \beta$-dimethyl-19-nortestosterone) made its debut recently in the field of male hormonal contraception. DMAU is an active androgen with progestational activity. Cleavage of the 17beta-ester bond by esterases in vivo gives rise to the biologically active androgen - dimetandrolone (DMA). Apart from androgenic activity of DMA, it also binds to the progesterone receptors and exerts progesteronic effect [19]. The DMA with its dual activity might have an important role as a single-agent oral contraceptive. Studies in rabbits have shown that the administration of DMAU at $2.5 \mathrm{mg} / \mathrm{kg} / \mathrm{d}$ resulted in the most effective contraceptive effect by reducing sperm count and sperm motility. Both low-dose $(1.0 \mathrm{mg} / \mathrm{kg} / \mathrm{d})$ and high-dose $(10.0 \mathrm{mg} / \mathrm{kg} / \mathrm{d})$ did not lead to infertility. Interestingly, the high dose did not show any harmful effects on sperm motility or sperm number [10]. The effect of DMAU is biphasic, but at an appropriate dose level exerts a contraceptive action.

\section{Connection Between Adverse Effects and Ways of Administration}

Testosterone is a key hormone for sexual behavior and sperm production. It affects social relations and regulates the psychophysical well-being. For this reason, birth control methods which affect hormonal balance should be precise enough and meet the requirements for the effective application of these methods. One factor affecting the acceptance in patients is the need to routinely inject the drug. The solution to this problem might be to use testosterone and nestorone transdermal gels. Studies show that this route of administration of hormones is more accepted among patients with the efficacy of $88-89 \%$ among different ethnic groups and allows us to effectively achieve the sperm concentration to less than 1 million/mL with minimal side effects [13]. An optimal hormone administration method has not yet been identified. Long-acting depot injections would be the most desirable way. In addition to the injection procedure, it should be verified whether testosterone or a synthetic androgen should be an active ingredient. Low intrinsic androgen activity of testosterone makes it technically difficult to use it in the depot formulation, since rather high doses of steroids are needed. Likewise it is still an open question as to whether combining androgen with another antigonadotropic compound is really advantageous, because no convincing data on increased efficiency or less intense side effects have been presented. Promising results were obtained using a combination of a progestin with testosterone esters in a delayed release formulation and administered by injection. According to present knowledge the optimal solution is subcutaneous implant of progestin and androgen [17].

\section{Non-Hormonal Male Contraception}

\section{Methods Disabling Sperm Motility}

Sperm motility has a significant impact on fertility and it became one of the targets for researchers developing male contraceptives. Studies have shown that the glycolytic pathways in mammalian sperm contain a number of isoenzymes that are not expressed in other cells of the body [20]. One of these enzymes is a germ cell-specific GAPDH (glyceraldehyde 3-phosphate dehydrogenase) that is expressed exclusively in spermatocytes and it is closely associated with the fibrous sheath that is part of the flagellum [27]. Studies in mice have demonstrated that GADPH is required for male fertility. The deficient enzyme activity and significantly lower ATP levels causes impaired sperm function that is observed as lack of progressive motility [21]. Mammalian spermatozoa have motile activity during ejaculation, but before reaching the egg they become hyperactivated in order to get through cumulus and zona pellucida. These movements require influx of calcium into the sperm through CatSper - voltage-gated ion channel located in the principal piece of the flagellum. Studies in mice have shown that targeted disruption of the gene encoding this channel results in male sterility. The motility of spermatozoa was decreased and they were unable to fertilize intact eggs [22]. 
These properties make CatSper channel an ideal target for both male and female contraception.

\section{Drugs Affecting Spermatogenesis}

Until now several substances can affect spermatogenesis, but none of them has yet been in clinical use. It is well known that vitamin A plays a significant role in spermatogenesis. Studies in mice have confirmed this thesis. RAR (retinoic acid receptor) is part of a signal transduction pathway modulated by vitamin A. RAR-null animals are infertile due to multiple spermatogenic defects [23]. BMS-189453 is an antagonist of all three types of retinoic acid receptors that has been created for patients suffering from skin and inflammatory diseases. Experiments conducted on rats have shown that one of its side effects is infertility. The disputed issue relates to the durability of this effect, but nevertheless there have been studies performed indicating that infertility in mice after oral administration of BMS-189453 is reversible [24].

\section{Drugs Suppressing Muscle Contraction of Vas Deferens}

Phenoxybenzamine, non-selective-adrenergic blocker, causes inhibition of ejaculation without affecting the libido, erection or orgasm. The effect is achieved by blocking the contractions of the longitudinal but not circular muscle of the vas deferens [25].

Another drug affecting vas deferens muscles contraction is thioridazine - an antipsychotic phenothiazine derivative [26].

\section{Lonidamine Analogues}

Adjudin is an analogue of an anticancer drug called lonidamine. The drug acts by blocking the maturation of spermatozoa in testicles by disabling the connection between Sertoli cells and young gametes. It's a completely reversible contraceptive effect was discovered in the 1980s. Fertility returns in rats after 11 weeks of drug withdrawal. Studies in dogs and rabbits also confirm this effect. However, the widespread use of it is excessively difficult because of nephrotoxicity, a major disadvantage that occurs with high dose intake.

Promising results were achieved with a modified connection of adjudin with FSH hormone without intrinsic activity, but still recognized by the specific receptors located in the Sertoli cells. This allowed for a significant reduction in the effective dose of adjudin and hence organ toxicity. Production costs of such preparation at the moment are too expensive to be widely used as contraception.
Other lonidamine analogues, gamendazole and CDB-4022, exert their effects by disrupting germ cell anchoring junctions in the seminiferous epithelium. Despite the promising effects of gamendazole, data of the acute and subchronic toxicity is unknown. Unlike gamendazole, CDB-4022 was well tolerated with no observable side effects. Perhaps this group of substances could be the future of male contraception, but still requires many years of research $[27,28]$

\section{Epidydymal Protease Inhibitor - Contraceptive Vaccine}

In 2004, Eppin (epididymal protease inhibitor) made its debut in the field of male contraception. Infertility was achieved by blocking the function of Epp (epididymal protease) in nonhuman primates using antibodies and it turned out to be reversible $[2,29]$. A study in small animals was conducted to assess the potential of Eppin as a contraceptive vaccine. Seven out of 9 male monkeys (78\%) developed high anti-EPP antibodies titers and were found to be infertile. Fertility was restored in 5 of the 7 monkeys (71\%) [29]. Epp is an epididymis-specific protein which functions as a central hub for a sperm surface protein complex. It exerts its effect by binding to SEMG1 (semenogelin-1) protein responsible for the coagulation of the semen in human spermatozoa to modulate the motility [30].

\section{Brdt Bromodomain Inhibitors - New Look at Male Contraception}

JQ1 blocks the production of sperm in the testes by blocking BRDT (Bromodomain testis-specific protein). The researchers found this bromodomain inhibition as a new therapeutic strategy in the male contraceptive field. Bromodomains are small modules that bind acetylated lysines. They are always part of a larger protein complex composed of many bromodomains and play a role in the regulation of gene transcription and chromatin remodeling [31]. The human genome is known to encode 56 bromodomains spread across 42 proteins [32]. The study of the spatial structure of bromodomains has shown that they have a common left-handed super-coil consisting of 4 antiparallel alpha-helices.

The acetylated-lysine binding site is located at the end of helix bundle and contains a conserved asparagine-containing chain segment, and tyrosine. The specificity and affinity of histone 
proteins recognition depends on $\mathrm{ZA}$ and $\mathrm{BC}$ loops surrounding the lysine-binding site and is different for each bromodomain [32].

The starting point for bromodomains research in the context of contraception was the existence of triazolothienodiazepine (JQ1) - a potent inhibitor of Brd4 bromodomain (Bromodomain-containing protein 4) that has a role in the pathogenesis of cancer by inhibiting the transcription of the oncogene Myc (myelocytomatosis oncogene) [34]. Blocking bromodomains in proteins involved in the transcription's initiation and elongation of other MYC-dependent genes leads to the cessation of cell cycle and division of tumor cells such as multiple myeloma or certain hematologic malignancies (AML-acute myeloid leukemia) [34]. Intensively proliferating germ cells have functional similarities to the tumor cells. Spermio- and spermatogenesis are unique processes in which the male genome must be properly packed to preserve its integrity during complex changes in the structure of chromatin and the entire developing germ cells. These phenomena require sophisticated control system of which bromodomains are part [32]. Dynamic structural changes in chromatin that occur during spermatogenesis are still not fully understood but it is clear that the correct Brdt is necessary for the proper conduct of these processes. Chromocenter is the central organizing chromatin structure of spermatids and mature sperm, and without the proper functioning of Brdt, it is impossible to achieve the correct architecture of the sperm nucleus [32]. The significance of bromodomains in spermatogenesis was inspired by the suspicion of their involvement in the pathogenesis of male infertility. In the research of Barda et al., 120 men $(n=120)$ with confirmed azoospermia, histological and cytological tests of testicular tissue were performed. Findings were as follows: a mixed cell atrophy, spermatocytes maturation arrest, SCO (Sertoli cell only), or normal spermatogenesis. The resulting formulations rated qualitatively and quantitatively bromodomain expression and the localization of four BET family genes as well as BRDT protein localization in testis and sperms. Brdt gene was not expressed in testicular tissue in patients with SCO leading to azoospermia, while the expression of the other three BET family bromodomains was invalid in patients with other causes of azoospermia [35]. Brdt protein was localized in testicular tissue, spermatocytes, spermatides and ejaculated spermatozoa. Brdt Protein expression was almost completely halted in testicular tissue samples from patients with impaired maturation of sperm. Importantly, the pattern of Brdt expression in humans differs from Brdt expression in mice. In humans Brdt is the only BET family member confined only to the expression of germ cells in the testes [35]. Brdt (ttestis) is a protein characteristic of testicular tissues associated with chromatin. Its expression occurs in spermatocytes (diplotenie, pachytenie) and round spermatides [36].

During postmeiotic maturation Brdt organises hiperacetylated histones in the nucleus binding to tetra-acetylated histone tail end 4 (H4Kac4) [37]. Genetic research of Brdt showed that the selective removal of the BRDT1 coding region is insufficient to obtain the effect of infertility [36]. This fact can be confirmed by the nucleotide polymorphisms of the Brdt gene, which is significantly associated with oligospermia or azoospermia in European men with idiopathic infertility [38]. All of these findings directed our attention to Brdt and Brd4 control as a potential target for male contraception. BRDT1 and BRD4 bromodomain structure have $81 \%$ identity and $89 \%$ similarity including the entire surface of contact with the inhibitor JQ1. Based on these similarities and the results of fluorimetry [33] it was decided to further investigate the influence of the JQ1 on BRDT1. It was found that direct Brdt inhibition by JQ1 shows a dose- and time of application-dependent effect of braking spermatogenesis. Structural studies of connection between JQ1 and Brdt exhibit complementarity type of ligand-receptor and complete closure of the acetylated lysine recognition site. In vivo JQ1 factor leads to impaired spermatogenesis and reduced sperm motility by affecting the meiotic and postmeiotic stages of development of male reproductive cells.

The macroscopic impact of the JQ1 in mice exposes itself as a reduction in the size of the testicles in all males after JQ1 exposure. Daily intraperitoneal injections of $50 \mathrm{mg} / \mathrm{kg}$ of JQ1 in male mice from the age of 3-6 weeks obtained a testis average size reduction of $75.4 \%$ testis sizes of the control group. In males of 6-9 weeks of age average reduction was $54.7 \%$ of testis size in relevant age control group. JQ1 administration for six weeks reduced the testicular volume to $40.6 \%$ of the testicles sizes in the control group. Thus, longer administration of the drug causes a greater reduction in testicular volume [31]. Researchers measured the impact of JQ1 treatment on levels of FSH, LH and testosterone because the absence of glycoprotein hormones and damage of androgen signal pathways causes male infertility [39]. Differences between the levels of these hormones in those exposed to the JQ1 group and the control group were not statistically significant. The weight of the seminal vesicles as the main androgen sensitive tissue was similar in both groups. In the mice treated with JQ1 the presence of normal, 
testosterone-producing Leydig cells was histologically confirmed. It follows from this that the agent JQ1 is germ cell-specific and does not affect hormonal-dependent processes [31]. Histological examination showed JQ1-dependent decrease in the number of tubules containing round spermatids and sperm in their light. Detailed microscopic analysis showed degeneration of the seminiferous tubules, and germ cell death, with multinucleated symplasts formation. These results are similar to those observed in the mice with knockout Ccnal (cycline1) gene, which are infertile due to a block in spermatogenesis [31]. In order to investigate the effect on sperm production, assayed for mouse epididymal spermatozoa in JQ1 test subjects. Sperm count decreased to $11 \%$ of the control group after 6 weeks of treatment. Analysis of sperm motility after 3 weeks of JQ1administration ( $50 \mathrm{mg} / \mathrm{kg}$ once daily) revealed 4.5-fold reduction in sperm motility. After six weeks of daily administration of JQ1 only $5 \%$ sperm showed increased motility as compared to $85 \%$ of sperm in the control group [31]. Twice larger doses of JQ1 caused damage effects similar to those observed in BRDT1 deficient mice research of Shang et al. [36].

Under the influence of JQ1 the expression of many genes essential to the germ cells is suppressed. These results are important because the lack of Cyclin A1 results in spermatocytes block in mice. Msy2 knockout mice are infertile with a block of postmeiotic cells and the lack of protein AKAP4 (A-kinase anchor protein 4) impairs motility. Lack of kinase $\mathrm{C}$ results in a polyploid nuclei as a result of blockage of meiosis [31]. In addition to the genes Ccna1 (cyclin A1), Msy2, Plk1 (pololike kinase 1) Aurkc (arora kinase C) and Akap4, other key genes expressed during meiosis or spermiogenesis, including Brdt, Papolb (poly (A) polymerase beta (testis specific)), Klf17 (Kruppel-like factor 17) and Prm1 (Protamine 1) are from 2.1 to 7.3 times lower in the testes of mice treated JQ1 compared with the control group [31]. In Brd4-dependent carcinomas, the antimitotic effect of JQ1 is related to the suppression of the cell cycle in the G1 phase. The possible nonspecific toxic effect on other tissues subject to strong proliferation, such as bone marrow or the large intestine, was not found. To exclude antimitotic effect via BRDT blockage researchers performed the phosphorylated serine 10 marking of histone $\mathrm{H} 3$, which develops during the condensation of the chromatin mitotic nuclei of spermatogonia. Changes in the content of this serine were not found, confirming the selective JQ1 effect on spermatocytes. Further confirmation has been found in the quantitative microscopic assessment of cells not recording a decrease of cyclin D1 (cell cycle regulator) in the nuclei of cells, and no increase in the number of Tunnel positive cells (Tunnel-test for locating DNA fragmentation). All performed measurements exclude non-specific antiproliferative effects of JQ1 toward BET bromodomains in mitotic germ cells [31].

In order to investigate the reversibility of the contraceptive effect after JQ1 withdrawal experiments taking into account the number of reproduction, the number of sperm and testicular weight were performed in mice after discontinuation of treatment. In mice treated with JQ1, the full effect of contraceptive sustained for one month after discontinuation of the drug, confirming the durability of action on spermatogenesis. After 4 months of JQ1 withdrawal all males spawned offspring in the number statistically similar to the control group. The average time to effective copulation after discontinuation of therapy was determined to $31.7 \pm 6.0$ days (26-38 days range). After complete mating period of 7 months (i.e. 4 months after discontinuance of administration of smaller doses of JQ1), the size of the testes, vas deferens, the surface of the seminiferous tubules, the histological construction of testicular sperm motility and number returned to levels statistically similar to the control group and were associated with the full fertility recovery [31]. Mice after JQ1 treatment at doses of $50 \mathrm{mg} /$ $/ \mathrm{kg} /$ day followed by $75 \mathrm{mg} / \mathrm{kg} /$ day regained fertility within 6 weeks after administration of the drug has been halted and the drug did not exhibit any long-term effect on fertility after its withdrawal.

For administration of JQ in higher doses and for longer periods in mice ( $50 \mathrm{mg} / \mathrm{kg}$ twice a day), the average time for effective mating after the discontinuation of therapy was determined to $65.7 \pm 7.7$ days (range: 58-81 days). Thus JQ1 prolonged therapy and/or increasing doses of the drug prolonged the infertility period for about one month. To more precisely determine the long-term effects of JQ, mouse testicles were examined after 2 and 4 months after 6 weeks discontinuation of JQ1 treatment at doses of $50 \mathrm{mg} / \mathrm{kg} /$ day. After 2 months, the number and motility of sperm increased in order to achieve normal parameters in four months. After this time seminoferous tubules were indistinguishable to control group [31]. Mice treated with high doses of JQ1 $(2 \times 50 \mathrm{mg} /$ $/ \mathrm{kg} /$ day) recovered the normal sperm number and motility after 60 days with confirmed fertility. Depending on the duration and total dose of JQ1, testis and sperm parameters were normalized within $1-3$ months leading to the recovery of full fertility [31].

External, pharmacological interference with the processes associated with the genetic material can, however, raise doubts about the safety and integrity of the information stored in it. The risk 
to offspring, resulting from DNA damage, would surely disqualify this type of contraception, but the results of studies in mice did not show such effects. JQ1 treated male's offspring showed a normal size, activity and behavior of a control group's offspring. The second generation's offspring of males treated with JQ1 also showed normal size and proper parameters of testicles and sperm. The impact of JQ1 on other members of the BET family proteins that are also expressed in testes of mice cannot be ruled out, but the state of mitotic spermatogonia remains unchanged in mice treated with JQ1. Other toxic effects on testicles or other tissues of mice such as mucosa of the gastrointestinal tract, which could indicate an antimitotic effect as a result of inhibition of Brd4 was not found [31]. JQ1 is clearly an inhibitor of BET family proteins and its primary contraceptive effect on germ cell line falls on a peak torque of BRDT protein expression.

The idea of blocking bromodomains as male contraception reinforces the belief that they are an easily available target. It should be emphasized that the studies on Brdt inhibitors are also carried out in other species and in future tests will be performed on humans. New derivatives of JQ1 with enhanced specificity for Brdt are created to increase the selectivity of the drug. Greater affinity and specificity will prevent long-term side effects of non-specific inhibition of other BET domains. To sum up, JQ1 appears to be the leading example of a new generation of drugs, which by inhibiting bromodomain during spermatogenesis, impairs the formation of sperm and their motility, leading to the reversible contraceptive effect without affecting the endocrine pathways.

\section{Juno and Izumo 1}

The recent discovery of an extracellular receptors pair essential for successful fusion of gametes set a context for the development of newer contraceptives. Gene disruption studies indicated that IZUMO1, a 377 amino acid type I transmembrane protein, is localised on spermatozoon but its receptor on egg remained unknown [40]. Recently, folate receptor 4 (Folr4) has finally been verified as the Izumol receptor on the mouse egg.
Researchers proposed to rename it Juno (after the Roman goddess of fertility and marriage) because of its effect on fertilization and its inability to bind folate. The Izumo-Juno interaction is conserved within several mammalian species, including humans and can be inhibited by an anti-Juno monoclonal antibody [40].

\section{Conclusions}

The situation of men in the field of contraception may change. This article reviews the presently available, soon to be available, and possible methods based on much more sophisticated knowledge of spermatogenesis, insemination and fertilization at the molecular level. Not every birth control method is mentioned above, only the main objectives for the development of a male contraceptive. The most popular form of contraception will be the result of the interaction of a number of medical and social factors, which vary in importance for one man to another. The currently available male birth control options should meet different expectations (as well as female options). Proven safety and simplicity of use of female contraception sets a high standard of excellence which it will be hard to equal in male contraceptive field. In addition to the possibility of reversing infertility, it should be affordable and, most of all, have the least side effects. A short description of the main methods indicate that different types of changes in the appearance and functioning of organism may occur. In some cases, the risk is likely to be disproportionate to the benefits and ideas surely will be abandoned, while in other cases developing the ideas will be possible because of potential benefits, that will be greater than potential risks. Taking birth control pills at the same time every day or doctor visits will be the duty of men who want to maintain contraceptive effectiveness and safety. To facilitate this task the new formulations should have a simple dosing schedule. The results of subsequent clinical trials verify which one of these methods will be most beneficial to the majority and which one will be a tool for men to level the playing field with women.

\section{References}

[1] Bremner WJ: Contraception for men: a breakthrough new approach. Cell 2012, 150, 667-668.

[2] Hoesl CE, Saad F, Pöppel M: Reversible, non-barrier male contraception: status and prospects. Eur Urol 2005, 48, 712-722.

[3] Zdrojewicz Z, Świerczyńska E: Prezerwatywy - historia i teraźniejszość. Seksuol Pol 2009, 7, 28-34.

[4] Cook LA, Van Vliet H, Lopez LM: Vasectomy occlusion techniques for male sterilization. Cochrane Database Syst Rev 2007, 18, CD003991.

[5] Thomas AJ Jr, Pontes JE, Rose NR: Microsurgical vasovasostomy: immunologic consequences and subsequent fertility. Fertil Steril 1981, 35, 447-450. 
[6] Belker AM: Microsurgical vasectomy reversal. In: Advances in Urology. Eds.: Lytton B, Catalona WJ, Lipshultz LI, McGuire EJ. Year Book Medical Publishers, Chicago 1988, 193-230.

[7] Alderman PM: The lurking sperm. A review of failures in 8879 vasectomies performed by one physician. JAMA $1988,259,3142-3144$.

[8] Linnet L, Hjort T, Fogh-Andersen P: Association between failure to impregnate after vasovasostomy and sperm agglutinins in semen. Lancet 1981, 1, 117-119.

[9] Practice Committee of American Society for Reproductive Medicine. Vasectomy reversal. Fertil Steril 2008, 90, 78-82.

[10] Attardi BJ, Engbring JA, Gropp D: Development of Dimethandrolone 17 $\beta$-Undecanoate (DMAU) as an Oral Male Hormonal Contraceptive: Induction of Infertility and Recovery of Fertility in Adult Male Rabbits. J Androl $2011,32,530-540$.

[11] Contraceptive efficacy of testosterone-induced azoospermia in normal men. World Health Organization Task Force on Methods for the Regulation of Male Fertility. Lancet 1990, 336, 955-959.

[12] Ilani N, Liu PY, Swerdloff RS: Does ethnicity matter in male hormonal contraceptive efficacy? Asian J Androl 2011, 13, 579-584.

[13] Ilani N, Roth MY, Amory JK: A New Combination of Testosterone and Nestorone Transdermal Gels for Male Hormonal Contraception. J Clin Endocrinol Metab 2012, 97, 3476-3486.

[14] Attardi BJ, Koduri S, Hild SA: Relative progestational and androgenic activity of four progestins used for male hormonal contraception assessed in vitro in relation to their ability to suppress LH secretion in the castrate rat. Mol Cell Endocrinol 2010, 328, 16-21.

[15] Herbst KL, Coviello AD, Page S: A single dose of the potent gonadotropin-releasing hormone antagonist acyline suppresses gonadotropins and testosterone for 2 weeks in healthy young men. J Clin Endocrinol Metab 2004, 89, 5959-5965.

[16] Huhtaniemi I, Nikula H, Rannikko S: Treatment of prostatic cancer with a gonadotropin-releasing hormone agonist analog: acute and long term effects on endocrine functions of testis tissue. J Clin Endocrinol Metab 1985, 61, 698-704.

[17] Pasqualotto FF, Lucon AM, Pasqualotto EB: Trends in male contraception Rev Hosp Clin Fac Med S Paulo 2003, $58,275-283$

[18] Walton MJ, Kumar N, Baird DT: 7alpha-methyl-19-nortestosterone (MENT) vs. testosterone in combination with etonogestrel implants for spermatogenic suppression in healthy men. J Androl 2007, 28, 679-688.

[19] Attardi BJ, Hild SA, Reel JR: Dimethandrolone undecanoate: a new potent orally active androgen with progestational activity. Endocrinology 2006, 147, 3016-3026.

[20] Krisfalusi M, Miki K, Magyar PL: Multiple glycolytic enzymes are tightly bound to the fibrous sheath of mouse spermatozoa. Biol Reprod 2006, 75, 270-278.

[21] Miki K, Qu W, Goulding EH: Glyceraldehyde 3-phosphate dehydrogenase-S, a sperm-specific glycolytic enzyme, is required for sperm motility and male fertility. Proc Natl Acad Sci U S A 2004, 101, 16501-16506.

[22] Ren D, Navarro B, Perez G: A sperm channel required for sperm motility and male fertility. Nature 2004, 413, 603-609.

[23] Chung SS, Wang X, Wolgemuth DJ: Male sterility in mice lacking retinoic acid receptor alpha involves specific abnormalities in spermiogenesis. Differentiation 2005, 73, 188-198.

[24] Chung SS, Wang X, Roberts SS: Oral administration of a retinoic acid receptor antagonist reversibly inhibits spermatogenesis in mice. Endocrinology 2011, 152, 2492-2502.

[25] Amobi NI, Smith IC: Differential inhibition in the human vas deferens by phenoxybenzamine: a possible mechanism for its contraceptive action. J Reprod Fertil 1995, 103, 215-221.

[26] Greenberg HR, Carrillo C: Thioridazine-induced inhibition of masturbatory ejaculation in an adolescent. Am J Psychiat 1968, 124, 991-993.

[27] Mok KW, Mruk DD, Lie PP: Adjudin, a potential male contraceptive, exerts its effects locally in the seminiferous epithelium of mammalian testes. Reproduction 2011, 141, 571-580.

[28] Zdrojewicz Z, Pietrusiewicz M, Markulak K: The male contraceptives: What's out there? Fam Med Prim Care Rev 2011, 13, 813-820.

[29] O’Rand MG, Widgren EE, Sivashanmugam P: Reversible immunocontraception in male monkeys immunized with eppin. Science 2004, 306, 1189-1190.

[30] Silva EJ, Hamil KG, Richardson RT: Characterization of EPPIN's semenogelin I binding site: a contraceptive drug target. Biol Reprod 2012, 87, 56.

[31] Matzuk MM, McKeown MR, Filippakopoulos P: Small-molecule inhibition of BRDT for male contraception. Cell 2012, 150, 673-684.

[32] Berkovits BD, Wolgemuth DJ: The first bromodomain of the testis-specific double bromodomain protein Brdt is required for chromocenter organization that is modulated by genetic background. Dev Biol 2011, 360, 358-368.

[33] Chung CW, Coste H, White JH: Discovery and characterization of small molecule inhibitors of the BET family bromodomains. J Med Chem 2011, 54, 3827-3838.

[34] Delmore JE, Issa GC, Lemieux ME: BET bromodomain inhibition as a therapeutic strategy to target c-Myc. Cell 2011, 146, 904-917.

[35] Barda S, Paz G, Yogev L: Expression of BET genes in testis of men with different spermatogenic impairments. Fertil Steril 2012, 97, 46-52. 
[36] Shang E, Nickerson HD, Wen D: The first bromodomain of Brdt, a testis-specific member of the BET sub-family of double-bromodomain-containing proteins, is essential for male germ cell differentiation. Development 2007, 134, 3507-3515.

[37] Morinière J, Rousseaux S, Steuerwald U: Cooperative binding of two acetylation marks on a histone tail by a single bromodomain. Nature 2009, 461, 664-668.

[38] Aston KI, Krausz C, Laface I: Evaluation of 172 candidate polymorphisms for association with oligozoospermia or azoospermia in a large cohort of men of European descent. Hum Reprod 2010, 25, 1383-1397.

[39] Matzuk MM, Lamb DJ: The biology of infertility: research advances and clinical challenges. Nat Med 2008, 14, 1197-1213.

[40] Bianchi E, Doe B, Goulding D: Juno is the egg Izumo receptor and is essential for mammalian fertilization. Nature 2014, 508, 483-487.

\section{Address for correspondence:}

Zygmunt Zdrojewicz

Department of Endocrinology, Diabetes and Radionuclide Therapy

Wroclaw Medical University

Pasteura 4

50-367 Wroclaw

Poland

Tel.: +48717842554

E-mail: zygmunt@zdrojewicz.wroc.pl

Conflict of interest: None declared

Received: 12.11.2014

Revised: 18.02.2015

Accepted: 23.02.2015 